\title{
Educational Planning Based on Prospective Scenarios in Distance Education
}

http://dx.doi.org/10.3991/ijes.v2i1.3673

J.C.C. Ribas, Federal Institute for Education, Science and Technology of Santa Catarina, Florianópolis, Brazil

Abstract-This study aims to analyze, within the realm of distance education, the contributions of the method for the construction and analysis of prospective scenarios applied to educational planning, proposed by Juares Thiesen. One of the approaches and techniques used by organizations to envision and design the long-term reality, which can contribute to overcome planning difficulties in distance education, are Prospective Studies and Scenario Building. The study, which was conducted by quantitative and qualitative approaches, was developed by the technical of research-action, organized into five stages: exploration, adaptation, implementation, analysis, and results. The work foresees the application of a unique method in real situation, through workshops. The verification of consistency and analysis of contributions of the method were conducted by questionnaire and interview at a public institution of higher education. The study highlights the importance of the method for construction and analysis of prospective scenarios in distance education and demonstrates how planning based on future scenarios contributes to the generation of an effective strategy, extrapolating trends and considering the future aspects and the uncertainties. It also represents a technical and scientific contribution, because in addition to investigating the application of a unique method, it presents the specificities and needs of its application for distance education.

\section{Planejamento Educacional Baseado em Cenários Prospectivos na Educação a Distância}

\author{
J.C.C. Ribas \\ Instituto Federal de Educação, Ciência e Tecnologia de Santa Catarina, Florianópolis, Brasil
}

\begin{abstract}
Resumo-Este trabalho tem por objetivo analisar, no âmbito da educação a distância, as contribuições do método para construção e análise de cenários prospectivos aplicado ao planejamento educacional, proposto por Juares Thiesen. Uma das abordagens e técnicas utilizadas pelas organizações para antever e projetar a realidade a longo prazo e que pode contribuir para a superação das dificuldades de planejamento da EaD, são os estudos prospectivos e a construção de cenários. $O$ estudo de cunho quantitativo e qualitativo foi desenvolvido por técnica de pesquisa-ação, organizada em cinco etapas: exploratória; adaptação; aplicação; análise; e resultados. Prevê a aplicação de um método singular em situação real, por meio de oficinas temáticas. A verificação de consistência e a análise das contribuições do método são realizadas por questionário e entrevista em uma instituição pública de ensino superior. $O$ estudo evidencia a importância para construção e análise de cenários prospectivos no âmbito da EaD e demonstra como o planejamento baseado em cenários prospectivos contribui para a geração de uma estratégia efetiva, extrapolando as tendências e considerando os aspectos do futuro e as incertezas. Constitui ainda uma contribuição técnica e científica, pois, além de investigar a aplicação de um método singular, apresenta as especificidades e necessidades de sua aplicação para a EaD.
\end{abstract}

Palavras-chave-Cenários prospectivos, educação a distância, planejamento, planejamento educacional,

\section{INTRODUÇÃO}

Preparar indivíduos competentes, não só visando a elevação da produtividade e competitividade, mas também a consolidação da democracia e maiores níveis de justiça social parece ser o grande desafio colocado para as instituições de ensino, especialmente para as instituições com oferta não paga, ou as chamadas instituições de ensino público no Brasil. Nesse sentido, a discussão sobre a relação entre os objetivos e resultados dos sistemas educacionais e a elevação da qualidade do setor produtivo ganham contornos cada vez mais amplos, sendo incorporada às agendas de diversos setores da sociedade.

Para as instituições de ensino público do Brasil, o enfrentamento desse desafio é especialmente urgente e estratégico. Principalmente quando se considera as atuais condições de oferta e a elevada demanda por cursos em todos os níveis no ensino público, tendo em vista a dívida social acumulada por muitos anos.

Recorrer a novas formas de ensino e programas dentre os quais pode ser destacada as ofertas regulares de cursos na modalidade a distância parece ser um caminho inovador em busca desta inclusão social e da transformação da realidade educacional brasileira. A Educação a Distância surge "como uma alternativa com condições de atender de forma eficaz a grande demanda por educação formal e por educação continuada" [1].

A adoção de planejamento visando atender a necessidade de dar aos projetos efetividade é um elemento importante para a conquista dos objetivos organizacionais. Dedicar tempo para que esse compartilhamento seja uma realidade é uma ação irrenunciável para os gestores organizacionais e, ao mesmo tempo, um desafio a ser vencido.

Entretanto, o planejamento da educação a distância tem constituído um problema, devido à carência de adoção de mecanismos capazes de propiciar o planejamento, organização, capacidade de direção e de controle, como um elemento basilar para que as organizações atinjam seus objetivos. 


\section{SPECIAL FOCUS PAPER \\ Planejamento Educacional Baseado Em CenÁRios Prospectivos na EduCAÇão a DistÂnCia}

O presente trabalho tem como objetivo apresentar os resultados obtidos em um projeto de pesquisa científica que investigou as contribuições dos estudos prospectivos como apoio ao planejamento educacional, no contexto da educação a distância. Para cumprir esse objetivo utiliza-se, o método proposto por Thiesen [2], para construção e análise de cenários prospectivos com foco no planejamento educacional.

\section{PLANEJAMENTO DA EAD EM CONTEXTO}

A educação tem se constituído em uma das grandes preocupações em todos os países e investimento nesta área representa o melhor caminho para a redução das desigualdades sociais e consequente melhora na qualidade de vida da sociedade.

Neste cenário, uma das principais possibilidades para atender a demanda de disseminação do conhecimento são as organizações e instituições educacionais que "buscam ampliar sua forma de ensino/aprendizagem, onde além de soluções presenciais, passam a incorporar, cada vez mais, a Educação a Distância (EaD)" [3]. Para [4], a Educação a Distância se apresenta como uma alternativa viável de atender a demanda por educação formal e continuada de maneira eficiente e eficaz.

Entretanto, apenas atender a uma demanda reprimida que cresce exponencialmente não basta, faz-se mister conhecer as necessidades e estabelecer as bases para um planejamento e gestão sólidos e vislumbrar o alcance possível para cada instituição. Estes são aspectos que não podem ser ignorados. Para [5], "fazer que todas as peças da engrenagem operem em um sistema de educação a distância exige um grau considerável de sofisticação gerencial, quase certamente mais do que em qualquer outro campo educacional".

Autores como [6], [7], [1], [8], [9], [10] entre outros, enaltecem a importância do papel da evolução das tecnologias de informação e comunicação (TIC's) aliadas ao crescimento da educação a distância e, a consequente necessidade de planejamento.

Mas por que planejar? [11] afirma que o planejamento estratégico abarca a empresa como um todo; envolve todos os recursos e áreas de atividade; preocupa-se em atingir objetivos em nível organizacional; é definido pela cúpula da organização e corresponde ao plano maior. $\mathrm{Na}$ educação a distância não é diferente.

Considerando o exposto, em uma modalidade de ensino como a $\mathrm{EaD}$, onde inúmeros relacionamentos se estabelecem nas instituições e nas interações necessárias em qualquer projeto, conhecer as necessidades e estabelecer as bases para um planejamento sólido e vislumbrar qual o alcance para a instituição e em maior análise para a sociedade, são aspectos determinantes.

Uma das abordagens e técnicas mais utilizadas pelas organizações para antever e projetar a realidade a longo prazo e que podem contribuir para a superação das dificuldades de planejamento da educação a distância são os estudos prospectivos ou estudos do futuro e a construção de cenários.

\section{ESTUDOS PROSPECTIVOS: TRAJETÓRIA E CONCEITOS}

Considerando a origem e desenvolvimento histórico dos estudos prospectivos, notadamente no tocante a ferramenta estratégica para planejamento de longo prazo, a literatu- ra faz referências a alguns fatos marcantes destacando que, embora a preocupação e o interesse em conhecer o futuro sejam antigos, a abordagem como metodologia prospectiva é relativamente recente.

As primeiras referências sobre os "planejadores do futuro" foram os profetas, presentes nas religiões judaica, cristã e islâmica. Na Grécia, o futuro era "predito" nos oráculos, locais onde adivinhos, sacerdotes e sacerdotisas realizavam a predição (discurso sobre uma condição futura). O mais famoso foi o Oráculo de Apolo, na antiga cidade grega de Delphos (o método Delphi, homenageia o oráculo de Delphos).

A construção histórica da prospectiva no século $\mathrm{XX}$ destaca diversas obras e eventos, assim como pesquisadores que ficaram conhecidos por seus métodos consagrados para a construção de cenários prospectivos, tais como: Michael Porter, Michel Godet, Peter Schwartz, Kees Van Der Heijden e Raul Grumbach, entre outros. Todos seguem os princípios descritos pela prospectiva e definem claramente as etapas para construção de cenários.

Em linhas gerais, podemos afirmar que a origem dos estudos prospectivos está relacionada com as experiências desenvolvidas na Europa, inicialmente pela França, com foco no planejamento público especialmente no campo das políticas sociais e nos EUA, com trabalhos mais direcionados para questões globais envolvendo as áreas de economia e segurança.

No que tange ao sentido da palavra prospectivo é evidente. Ela se opõe a retrospectivo, pois olhamos para frente e não para trás. "Um estudo retrospectivo examina o passado, enquanto que uma pesquisa prospectiva se dedica a estudar o futuro" [12].

Os estudos prospectivos constituem elemento importante do processo de planejamento, haja vista oferecem orientação para as tomadas de decisões sobre iniciativas e ações para a construção de um futuro almejado pelas instituições e consequentemente pela sociedade. A própria atividade planejadora tem como elemento balizador o fato de o futuro não estar predeterminado e ser uma construção social, resultante das ações e das decisões da sociedade. $\mathrm{O}$ processo de planejamento não teria nenhum sentido se a natureza e a sociedade tivessem histórias futuras já traçadas, eliminando qualquer espaço de liberdade para definir o próprio futuro [13].

As definições apresentadas pela literatura, de modo geral, colocam os estudos prospectivos ou estudos de futuro como categorias mais amplas que incorporam o conjunto de metodologias e técnicas, dentre elas a construção de cenários ou "cenarização". Esses estudos são voltados, essencialmente, às organizações privadas nos setores de serviços e tecnologia, sobretudo no campo da economia. Planejar por meio de métodos e técnicas de cenários prospectivos implica em definir estratégias que definirão a vida futura da organização, e, para tanto, estão disponíveis na literatura inúmeras ferramentas e metodologias.

\section{O MÉTODO PROPOSTO POR THIESEN}

Buscando reduzir o abismo entre os trabalhos de construção e análise de cenários com foco na educação e entre os voltados às atividades econômicas e industriais, Juares Thiesen, propôs em sua tese de doutoramento o desenvolvimento de um método para a construção e análise de cenários prospectivos em planejamento educacional baseado na Gestão do Conhecimento (GC). Sua proposta, de 


\section{SPECIAL FOCUS PAPER \\ Planejamento Educacional Baseado em CenÁRios Prospectivos na EduCAÇÃo a DistÂnCia}

cunho teórico e de alto nível de abstração, foi elaborada a partir do conjunto de metodologias e técnicas adotadas em estudos prospectivos nas diversas áreas da produção econômica, agregando teorias e práticas da Gestão do Conhecimento.

Este método foi construído a partir de propostas metodológicas de Michel Godet, Kess Van Der Heijden, Michel A. Porter, Raul Grumbach e Peter Schwartz. Além desse, outros trabalhos e autores também são citados, como: GBN (Global Business Network), a CEDEFOP (European Centre for the Development of Vocational Training), os trabalhos de P. J. H Shoemaker e J. Coates.

A tabela I apresenta um resumo do método proposto por Thiesen, caracterizando as fases e sua descrição. São previstas pelo método a utilização de inúmeras técnicas e ferramentas, tais como: workshops, método Delphi, brainstorming, análise estrutural de Godet, estruturação dedutiva de Kees Van Der Heijden, entre outras.

TABELA I.

FASES DO MÉTODO PROPOSTO POR JUARES THIESEN

\begin{tabular}{|c|l|}
\hline FASE & \multicolumn{1}{|c|}{ DESCRIÇÃO DA FASE } \\
\hline 01 & Construção da base para o trabalho \\
\hline 02 & $\begin{array}{l}\text { Construindo o diagnóstico do sistema de ensino e definindo } \\
\text { o problema central }\end{array}$ \\
\hline 03 & Compreendendo o jogo dos atores externos \\
\hline 04 & Identificando os fatos portadores de futuro \\
\hline 05 & Gerando cenários e testando sua consistência \\
\hline 06 & $\begin{array}{l}\text { Avaliando os cenários como decisão estratégica para a } \\
\text { elaboração do Plano de Desenvolvimento da Educação }\end{array}$ \\
\hline
\end{tabular}

\section{PESQUISA-AÇÃO}

De acordo com [14] o "planejamento de uma pesquisaação é muito flexível. Contrariamente a outros tipos de pesquisa, não segue uma série de fases rigidamente ordenadas".

Para atingir os objetivos desta pesquisa, as atividades desenvolvidas que caracterizam a utilização da técnica de pesquisa-ação, foram subdividas em cinco etapas: exploratória; adaptação; aplicação; análise; e resultados. A seguir, encontram-se detalhadas as etapas da pesquisa.

\section{Etapa 1 - Exploratória}

A primeira etapa, a exploratória, é composta pelos elementos que sustentam e justificam a presente pesquisa: o tema, o problema de pesquisa e os objetivos. Compõe ainda a etapa exploratória a organização da revisão bibliográfica para a fundamentação da pesquisa sobre os temas que servem de base para o trabalho desenvolvido. Nesta organização procede-se o estudo sobre os pilares que sustentam a presente pesquisa: os estudos prospectivos, a educação a distância e o método para construção e análise de cenários prospectivos aplicado ao campo educacional proposto por Thiesen.

\section{Etapa 2 - Adaptação}

A etapa de adaptação visa à análise aprofundada do método proposto por Thiesen para construção e análise de cenários prospectivos, com foco no planejamento educacional, com o objetivo de tornar sua aplicação possível, em ambiente acadêmico e situação real.

Analisando as etapas do método propostas pelo referido autor, observa-se o alto nível de abstração em sua proposição metodológica, refletindo etapas de relativa comple- xidade e utilização de ferramentas com elevado nível de sofisticação para aplicação em ambiente acadêmico, no qual restrições são identificadas, como por exemplo, a disponibilização de tempo para participação das oficinas para construção e análise dos cenários prospectivos e o número de oficinas necessárias.

Diante disso, e após análise criteriosa das etapas metodológicas que compõe o método em tela, procedeu-se à elaboração de um plano de adaptação da aplicação do método, mantendo sua orientação teórico-metodológica, preservando assim a essência do método.

Assim, foram introduzidas as seguintes adaptações no processo de construção dos cenários prospectivos, visando tornar possível e viável sua aplicação:

- inclusão da Ideia de Negócio de Kees Van Der Heijden: Essa estratégia promove uma visão holística da instituição e foi incluída com o objetivo de simplificar o processo de construção do diagnóstico do sistema de ensino e definição do problema central;

- Método Delphi e Brainstorming: O autor do método em questão enaltece a utilização dessas duas ferramentas de apoio à construção de cenários em distintas fases do método. Salienta o uso da técnica brainstorming de ajuda à criatividade por ser um processo de trabalho em grupo que visa produzir inúmeras soluções possíveis para determinado problema, além de estimular a imaginação e contribuir para o surgimento de ideias. Salienta também a aplicação do método Delphi para obter o consenso de opiniões de um grupo de pessoas;

- Ambientes de prospecção: O método proposto por Thiesen assinala a necessidade de geração dos cenários para posterior teste de consistência, em sua penúltima fase. Para tanto, sugere que o estudo resulte na construção de poucos cenários (entre 3 e 5). Privilegiando o trabalho coletivo, a simplificação de etapas e redução da complexidade, optou-se por determinar antecipadamente os cenários preferenciais a serem construídos: o cenário utópico, o cenário realista e o cenário de crise. Também foi definido o horizonte temporal para prospecção em oito anos. $\mathrm{O}$ fato de determinar inicialmente os ambientes a serem prospectados e horizonte temporal reduziu sobremaneira a complexidade do processo de construção, já que algumas ferramentas não foram utilizadas.

- Carga horária total e número de oficinas: Considerando a necessidade de tornar a aplicação do método possível e viável, adaptou-se a carga horária e o número de oficinas de maneira que o grupo de trabalho participasse integralmente de todas as atividades, evitando descontinuidade no processo de construção coletiva. Desta forma e considerando que o processo de construção de cenários ocorreu de maneira participativa e cooperativa em ambiente acadêmico e situação real, o número de oficinas definidas para realização do trabalho foi de quatro encontros, com carga horária inicialmente prevista de 3 horas cada oficina, totalizando doze horas.

\section{Etapa 3 - Aplicação}

A aplicação do método para prospecção de cenários na educação a distância, utilizado como referencial teóricometodológico, foi realizado em uma Instituição Pública de Ensino Superior (IPES), com um grupo de dez (10) atores 


\section{SPECIAL FOCUS PAPER \\ Planejamento Educacional Baseado Em CenÁRios Prospectivos na EduCAÇão a DistÂnCia}

de destacada atuação na EaD e prevê a realização de quatro oficinas com os sujeitos da pesquisa e um conjunto de atividades externas desenvolvidas por uma equipe de suporte metodológico.

A primeira oficina estabeleceu as bases para o trabalho e diagnóstico do sistema central. Foi desenvolvida em três momentos: o primeiro de sensibilização dos sujeitos da pesquisa, com apresentação de conceitos, em slides, sobre $\mathrm{EaD}$, estudos prospectivos e o método proposto por Thiesen. Em seguida foi proferida palestra sobre o tema "Planejamento por Cenários Prospectivos". O segundo momento foi destinado ao diagnóstico do sistema de ensino da EaD, interno e externo, e culminou com a construção da ideia de negócio da instituição, de acordo com a estratégia de Kess Van Der Heijden. O terceiro momento foi destinado a definição dos parâmetros utilizados para a construção dos cenários prospectivos: enfoque prospectivos; área de concentração EaD; horizonte temporal de 8 anos; cenários prospectados: realista, de crise e inovador.

A segunda oficina teve por objetivo definir as variáveis e atores da EaD que fariam parte do processo de construção dos cenários prospectivos. A partir de uma lista previamente elaborada de 20 variáveis e 12 atores, foi aplicado o método Delphi para atingir o consenso de ideias. Após duas rodadas de aplicação do Delphi, chegou-se as cinco variáveis (gestão; orçamento e finanças; infraestrutura de apoio; tecnologia; PDI/PPI/PPC) e quatro atores (alunos; professores; governo federal/MEC; reitoria/direção) mais relevantes para os sujeitos da pesquisa.

A terceira oficina visou a construção das relações e influências entre as variáveis $\mathrm{x}$ atores anteriormente definidos. Foram formados quatro subgrupos e utilizada a técnica de brainstorming para construção das relações e influências para cada um dos cenários de realidade, de crise e inovador. No momento seguinte houve a socialização dos trabalhos realizados por grupos menores ao grande grupo visam aperfeiçoar as propostas. Após a realização desta oficina, em trabalho externo, a equipe de suporte metodológico validou os cenários e elaborou os respectivos enredos.

A quarta oficina foi destinada para avaliação dos cenários como decisão estratégica para o planejamento educacional pelos sujeitos da pesquisa.

\section{Etapa 4 - Análise}

Foram previstos para esta etapa o teste de consistência e a análise das contribuições do método para construção de cenários prospectivos, que foram realizadas por aplicação de questionário (abordagem quantitativa) e entrevista (abordagem qualitativa), como instrumentos de coleta de dados.

Primeiramente foi aplicado o questionário com o intuito de coletar dados preliminares sobre o fenômeno em estudo, enviando um link para preenchimento disponível no Google Docs. Após tabulação e análise dos dados do questionário, passa-se para a fase das entrevistas, cujo objetivo é compreender e aprofundar o fenômeno.

O roteiro da entrevista, construído a partir da base conceitual teórica e documental, teve por objetivo coletar informações e compreender com maior profundidade o fenômeno em estudo em seu contexto, sendo classificada com uma entrevista estruturada, organizada em três blocos: o primeiro com questões relativas à consistência do método utilizado para construção e análise de cenários prospectivos com foco na educação, o segundo com vista à obtenção de dados no que se refere às contribuições do método ao planejamento da educação a distância e o terceiro buscando explorar as dificuldades no desenvolvimento das atividades da $\mathrm{EaD}$ e como a modalidade a distância contribui para o cumprimento da missão institucional.

Foi utilizado o software QualiQuantSoft [15], como organizador de dados, para manipulação das informações oriundas das entrevistas. Consistência do método, contribuições do método e dificuldades no exercício das atividades da EaD foram as categorias de análise consideradas.

\section{Etapa 5 - Resultados}

Após finalização das etapas de aplicação e análise, procede-se a análise geral desses resultados, sendo elaboradas as conclusões finais da pesquisa.

\section{ESPECIFICIDADES DO PROCESSO DE APLICAÇÃO DO MÉTODO}

Considerando as atividades de aplicação do método proposto na realização das quatro oficinas, a seguir apresentam-se as dificuldades, facilidades e recomendações.

\section{Dificuldades Encontradas}

Em relação às dificuldades encontradas, salientam-se os seguintes pontos:

- Processo de convencimento dos possíveis integrantes do grupo de trabalho, para participarem deste projeto de pesquisa;

- Estratégia para manutenção dos dez integrantes de grupo de trabalho nas quatro oficinas de construção dos cenários constituiu-se em tarefa árdua, anterior a todas as oficinas com telefonemas, e-mail e reforço das solicitações, pessoalmente;

- O método proposto por Thiesen, para construção e análise de cenários prospectivos voltados ao planejamento educacional, foi concebido e validado teoricamente por especialistas. A aplicação do método requereu adaptações, visando tornar sua aplicação, em situação real, viável e possível;

- Definição das ferramentas na etapa de adaptação da pesquisa-ação que foram utilizadas para simplificar e tornar factível a construção dos cenários, sem dispersar os componentes do grupo de trabalho. Definiu-se a técnica de brainstorming, o método Delphi, ideia de negócio, estruturação dedutiva de cenários e desenvolvimento de histórias, e o método de estruturação dedutiva de cenários e história dos enredos, como as mais apropriadas para o estudo, na EaD. Acredita-se que tais ferramentas também poderiam ser utilizadas na modalidade presencial ou outros ambientes educacionais;

- Percebeu-se que alguns integrantes do grupo de trabalho apresentaram níveis de conhecimento diferentes, causando alguma dificuldade para equalização de entendimento acerca do conteúdo abordado. Associado a isso, o fato de cada integrante terem um entendimento distinto do que é importante para a área em estudo, é significativo. Além disso, o entendimento sobre as relações de influência entre variáveis e atores também difere, conforme análise dos participantes de cada subgrupo de trabalho; 
- Esforço empreendido para manter o envolvimento dos elementos do grupo de trabalho, da primeira à quarta oficina. Apesar de inúmeros contatos antes das oficinas, duas pessoas não participaram integralmente do processo;

- Houve necessidade de reorganização das variáveis e atores, visando um resultado mais efetivo, sendo necessário reduzir o número de variáveis e atores para reduzir a complexidade das relações e influências estabelecidas;

- Percebeu-se a necessidade de um tempo maior para realização das atividades relativas ao exercício prospectivo, uma vez que desde a primeira oficina o tempo destinado às atividades foi restrito, sendo necessário limitar alguns processos reflexivos do GT.

\section{Facilidades Encontradas}

Considerando o processo de construção dos cenários, foram identificadas as seguintes facilidades:

- Definição dos sujeitos da pesquisa para realização dos estudos prospectivos, pois a maioria dos cursos ofertados na modalidade a distância, está vinculados ao departamento acadêmico ao qual este pesquisador também mantém vínculo, facilitando assim o contato com os colaboradores no processo;

- Clima cordial e de colaboração do grupo de trabalho com a equipe de suporte metodológico e vice-versa, durante a realização das oficinas, favoreceram o desenvolvimento das atividades;

- Palestrante externo no processo de sensibilização do GT, dado ao seu currículo e experiência, favoreceu o processo, observando-se após a palestra um grupo disposto e estimulado em participar do estudo;

- Inclusão da ferramenta de concepção da ideia de negócio que proporcionou uma visão holística da EaD, auxiliando os integrantes do grupo de trabalho na formação de visão ampliada sobre o passado, presente e as possibilidades de futuro da $\mathrm{EaD}$, no lócus do estudo;

- Utilização das ferramentas e técnicas definidas na etapa de adaptação da pesquisa-ação para simplificar e tornar factível a construção dos cenários;

- A elaboração de uma lista prévia de variáveis e atores e os respectivos conceitos, preparada e organizada pela equipe de suporte metodológico, facilitou a compreensão inicial do grupo de trabalho e agilizou o andamento da oficina;

- Facilidade para se chegar ao consenso do grupo de trabalho durante a realização das oficinas, o que confirma a convergência de interesses do grupo;

- Identificou-se que o envolvimento de grande parte dos sujeitos da pesquisa pode ser definido como "coeso e convergente", pois da primeira à última oficina, percebeu-se que o envolvimento dos atores foi participativo e estável.

\section{Recomendações}

Neste tópico, faz-se algumas recomendações a partir do processo de aplicação do método proposto, no contexto da $\mathrm{EaD}$ :

- Realizar oficina prévia para sondagem de possível integrante do futuro grupo de trabalho, que permita discutir, entender e caracterizar melhor os temas em estudo, para que todas as pessoas que demonstrarem interesse nivelem seus conhecimentos e despertem para as novas possibilidades em vista de um planejamento a longo prazo;

- Solicitar indicações de outros potenciais integrantes do grupo (especialistas, representantes de instituições, empresas) aos participantes da primeira oficina, para integrá-los ao grupo nas próximas etapas do estudo prospectivo com o propósito de ampliar o número de participantes e dar maior consistência ao processo de construção coletiva;

- Ampliar o tempo de duração das oficinas, propiciando maior espaço para interação entre os participantes;

- Na "Oficina 1", definir estratégias auxiliares, além da ampliação do tempo, para possibilitar que o tema e horizonte prospectivo, bem como os conceitos correlatos fiquem claros e que se permita seu amadurecimento, com o propósito de facilitar o entendimento dos participantes e acelerar as atividades da oficina. Dirigir toda e qualquer dúvida existente, bem como oferecer espaço para a manifestação dos integrantes do grupo de trabalho é fundamental para o sucesso da atividade, em especial na oficina inicial;

- Recomenda-se enviar tarefas previamente definidas para facilitar o desenvolvimento das oficinas e contribuir para um melhor amadurecimento do tema. A proposta de envio de material prévio, sob forma de, por exemplo, textos e artigos, levará a uma economia de tempo para o desenvolvimento das demais atividades;

- Dentro das possibilidades, realizar as oficinas em local que evite a dispersão dos integrantes do grupo de trabalho, durante e nos intervalos das mesmas;

- É conveniente, no primeiro momento de cada oficina, que um dos integrantes do grupo faça uma síntese das atividades desenvolvidas na oficina anterior;

- Trabalhar, sempre que possível, com um número reduzido e suficiente de variáveis e atores, visando facilitar e minimizar a complexidade do trabalho das relações e influências entre variável versus atores;

- A questão do consenso de ideias deve ser cuidadosamente conduzida, uma vez que, dependendo do perfil dos participantes do grupo de trabalho, de interesses múltiplos, pode acarretar na necessidade de ampliação do tempo inicialmente previsto para o desenvolvimento da oficina;

- Em outra aplicação desse método, para a EaD, prever a realização de uma ou mais oficinas para sensibilização, com o objetivo de manter os integrantes do grupo de trabalho envolvidos com o estudo prospectivo e $\mathrm{EaD}$, oferecendo-lhes a possibilidade de esclarecer pontos específicos.

\section{RESULTADOS OBTIDOS EM RELAÇÃO À APLICAÇÃO DO MÉTODO}

Faz-se algumas considerações em relação aos resultados obtidos pela aplicação do método proposto, sob dois olhares que constituem a sua essência: o poder de construção das oficinas e a perspectiva de planejamento para a $\mathrm{EaD}$. Em relação ao poder de construção das oficinas, destaca-se:

- O ambiente proporcionado pelas oficinas permitiu uma discussão de forma interdisciplinar tendo a par- 
ticipação de diversos atores num movimento de planejamento, sendo esse movimento uma carência observada na educação, em especial na educação a distância que vive um momento de definição, de construção em torno de sua institucionalização;

- As oficinas permitiram o estabelecimento de pontos de referência para o desenvolvimento de um planejamento, de uma visão de futuro, de onde se está e onde é possível chegar e o que precisa ser feito para atingir esses objetivos e quais adversidades podem ocorrer. As oficinas proporcionaram com esses novos pontos de referência, uma nova consciência frente aos aspectos que precisam ser trabalhados na educação a distância;

- A estruturação e sequência, as etapas, técnicas e ferramentas utilizadas nas oficinas contribuíram para que cada um dos participantes compreendesse o que significa aplicar o método e qual o impacto que esse método pode trazer para a mudança da realidade vivida no cotidiano da instituição;

Em relação à perspectiva do planejamento para $\mathrm{EaD}$, destaca-se:

- O método traz ao planejamento da educação a distância a organização de um trabalho de construção coletiva, por meio de uma ação de formação de grupos, na qual esses grupos passam a compreender, de forma integrada, que podem interferir diretamente nas práticas do dia a dia da instituição, e que essa compreensão pode gerar planos operacionais visando dar uma estrutura para o trabalho diário;

- O método em si traz uma nova perspectiva de maneira objetiva, consistente, com elementos de análise pontuais para que seja possível fazer reflexões, entender quais são as possibilidades de intervenção para que a realidade que se pretende seja construída com a participação dos vários atores envolvidos.

- A utilização do método aplicado ao planejamento da educação a distância trouxe uma perspectiva inovadora e fez com que os participantes pudessem entender o potencial da aplicação dos cenários prospectivos ao planejamento da EaD.

Assim, considerando os aspectos citados e após realizadas todas as atividades de pesquisa e análises, conclui-se que o método proposto apresentou-se eficaz quanto a sua consistência e contribuições para construção e análise de cenários prospectivos aplicados ao planejamento educacional, no âmbito da educação a distância, mesmo com as adaptações introduzidas para tornar sua aplicação possível e viável.

\section{RESULTADOS OBTIDOS EM RELAÇÃO À CONSISTÊNCIA E CONTRIBUIÇÕE DO MÉTODO}

Destacam-se ainda, considerações importantes em relação à consistência e contribuições do método, face ao desenvolvimento desta pesquisa, conforme segue:

- O método proposto por Thiesen e validado apenas teoricamente, foi amplamente avaliado, permitindo definição de suas etapas, ferramentas e técnicas visando tornar sua aplicação possível e viável, em situação real;

- Os cenários construídos pela aplicação do método proposto, com foco na área educacional, têm como essência o conhecimento construído no processo coletivo que relaciona claramente os aspectos considerados convergentes entre os conceitos de estudos prospectivos e educação a distância;

- A aplicação do método proposto para construção e análise de cenários prospectivos na educação a distância ocorreu conforme previsto e apontou a pertinência de seu uso, caracterizando-o como adaptado à realidade, simples e pragmático, avalizando sua eficácia;

- Aplicou-se o método para construção e análise de cenários prospectivos, no contexto da educação a distância, atingindo resultados positivos, tanto em termos da consistência do método como de suas contribuições ao planejamento educacional;

- As oficinas facilitaram, aos sujeitos envolvidos na pesquisa, a compreensão sobre a importância de elaborar um planejamento a partir de um trabalho de construção coletiva;

- As múltiplas experiências dos sujeitos da pesquisa na educação a distância, contribuíram significativamente para o desenvolvimento das atividades da pesquisa;

- A aplicação do método proposto, em situação real, na educação a distância, caracterizando uma aplicação empírica de um método singular, formulado e validado apenas no campo teórico, oferece subsídios para a academia e profissionais interessados em ampliar conhecimentos acerca do tema, e assim, contribuir com um campo amplo para pesquisa, podendo o método ser aplicado, com os devidos cuidados, nos diversos setores da educação;

- A construção de cenários apresenta-se como uma ferramenta de apoio à decisão eficaz sob a perspectiva do planejamento, oportunizando aos gestores abandonarem a postura meramente reativa (bombeiro), para adotarem a postura preventiva (prevenido) e aproveitar oportunidades passando a uma postura pró-ativa (conspirador), no que diz respeito ao planejamento da EaD.

\section{CONSIDERAÇÕES FINAIS}

Com os resultados obtidos com esta pesquisa, identifica-se sua relevância no campo teórico na medida em que utiliza um instrumento para apoio ao planejamento estratégico, preenchendo uma lacuna tanto na experimentação empírica do método proposto para construção e análise de cenários voltados ao planejamento educacional, como no planejamento estratégico baseado em cenários para a educação a distância.

Com os conhecimentos construídos, pretende-se contribuir técnica e cientificamente para com a necessidade da aplicação de um método singular, aplicável ao planejamento gestão da EaD. Acredita-se que a construção de cenários, é um modo inovador e opera nos processos de Gestão do Conhecimento, no sentido tanto do resgate dos conhecimentos explícitos como na apropriação e sistematização dos conhecimentos implícitos de modo interdisciplinar.

Este estudo, dada a originalidade do tema, pode contribuir para com o surgimento de novas metodologias, requerendo a continuidade da pesquisa e ampliando as produções acadêmicas, em especial no que tange ao planejamento e gestão da educação a distância. 


\section{SPECIAL FOCUS PAPER \\ Planejamento Educacional Baseado Em CenÁRios Prospectivos na EduCAÇÃo a DistÂnCia}

\section{REFERÊNCIAS}

[1] POLAK, Y. N. S; DUARTE, E. C. V.; ASSIS, E. M. Construindo do novo conceito da gestão de sistemas de educação a distância. In: 14 CIAED Congresso Internacional ABED de Educação a Distância, Santos/SP, 2008.

[2] THIESEN, J. S. Método para a construção e análise de cenários prospectivos em planejamento educacional baseado na gestão do conhecimento. Tese (Doutorado em Engenharia e Gestão do Conhecimento) - Universidade Federal de Santa Catarina, 2009.

[3] LENZI, G. K. S. Diretrizes para a gestão de projetos de cursos de capacitação na modalidade de Educação a Distância. Dissertação (Mestrado em Engenharia e Gestão do Conhecimento) - UFSC, 2010

[4] NUNES, I. B. A história da EAD no mundo. In Educação a Distância: o estado da arte. (Orgs.) LITTO, F.M.; FORMIGA, M. São Paulo: Pearson Education do Brasil, 2009.

[5] MOORE, M.; KEARSLEY, G. Educação a distância: uma visão integrada. São Paulo: Thompson Pioneira, 2007.

[6] CATAPAN, A. H. Mediação Pedagógica Diferenciada. In: ALONSO, Katia Morosov; RODRIGUES, Rosangela S; BARBOSA, Joaquim G. (orgs) Educação a Distância: práticas, reflexões e Cenários plurais. Cuiabá: EdUFMT, 2010 P 71-79

[7] MILL, D.; BRITO, N. D.; SILVA, A. R.; ALMEIDA, L. F. Gestão da educação a distância $(\mathrm{EaD})$ : noções sobre planejamento, organização, direção e controle da EaD. IFSJ. Revista Vertentes, n. 35,2010 .

[8] RIBAS, J. C. C., MOREIRA, B. C. M., Silva, E. R. G., BATTISTI, P., PEREIRA, A. T. C. Gestão de polo de apoio presencial: um desafio ao desenvolvimento da educação a distância no sistema Universidade Aberta do Brasil In: VII ESUD 2010 Congresso Brasileiro de Ensino Superior a Distância, Cuiabá: 2010.

[9] RIBAS, J. C. C., HERMENEGILDO, J. L. S. A implantação da Educação a Distância pelo Sistema Universidade Aberta do Brasil no CEFET-SC: Caminhos e percursos. In: TICAI (TICs Aplicadas a la enseñanza/aprendizaje de la Ingeniería), 2009

[10] SPANHOL, F. J. Aspectos do gerenciamento de projetos em EAD. In Educação a Distância: o estado da arte. (Orgs.) LITTO, F.M.; FORMIGA, M. Pearson Education do Brasil: São Paulo, 2009

[11] CHIAVENATO, I. Introdução a teoria geral da administração. Rio de Janeiro: Elsevier, 2004.

[12] BERGER, G. A atitude prospectiva. Tradução. Nathália Kneipp. Outubro/2004. In: Revista Parcerias estratégicas. Brasília/DF: Centro de Gestão e estudos estratégicos. N. 19, Dez/2004.

[13] GODET, M. Manual de prospectiva y estratégia: De la antecipación a La acción. Barcelona: Marcombo, 1993.

[14] THIOLLENT, M. Metodologia da pesquisa-ação. $18^{\mathrm{a}}$ edição. São Paulo: Cortez, 2011.

[15] LEFÈVRE, F. Discurso do Sujeito Coletivo (DSC). Principais conceitos. Disponível em: http://www.fsp.usp.br/qualisaude/Discurso_principais_conceitos.htm $>$. Acesso em: $13 \mathrm{dez}$ 2012.

\section{AUTOR}

Júlio César da Costa Ribas, é Doutor em Engenharia e Gestão do Conhecimento, Graduado e Mestre em Ciência da Computação pela Universidade Federal de Santa Catarina - UFSC; Licenciado em Informática pela Universidade Tecnológica do Paraná - UTFPR - e Especialista em Informática Aplicada a Educação pela Universidade Estadual de Campinas - UNICAMP. Professor e Analista de Tecnologia da Informação, do Instituto Federal de Santa Catarina, Florianópolis, Santa Catarina, Brasil, (email:julio@ifsc.edu.br).

Submitted 19 March 2014. Published as re-submitted by the authors 05 April 2014. 